\title{
ON THE MAINTENANCE OF GENETIC VARIATION BY DISRUPTIVE SELECTION AMONG HOSTS IN A PHYTOPHAGOUS MITE
}

\author{
JAMES D. FRY' \\ Department of Biology, University of Michigan, Ann Arbor, MI 48109 USA
}

Key words. -Genetic variation, mites, plant-herbivore interactions, selection, Tetranychus urticae.

Received February 12, 1991. Accepted April 11, 1991.

The phytophagous mite Tetranychus urticae is broadly polyphagous, having been reported from hundreds of different host species in many different families (Jeppson et al., 1975). Some hosts, such as varieties of tomato and cucumber, typically cause high juvenile mortality of $T$. urticae (e.g., Gould, 1978a, 1979; Fry, 1988a, 1989), even though these hosts are often used by natural populations of the mites (Gilbert et al., 1966; Gould, 1978b; Fry, 1988a). In laboratory experiments, however, $T$. urticae populations have been shown to be able to rapidly adapt to tomato, cucumber, and other initially unfavorable hosts, sometimes experiencing more than two-fold gains in survival in under 10 generations (Gould, 1979; Fry, 1989). This raises the question of how genetic variation for fitness on these hosts is maintained. In other words, why should populations often be far below their fitness maxima in environments they normally encounter?

One obvious way of answering this question is to invoke "trade-offs" in adaptation to different hosts (Gould, 1979; Rausher, 1983; Mitter and Futuyma, 1983; Futuyma and Peterson, 1985). If genotypes favored by selection on some hosts are selected against on others, this could keep populations from attaining their fitness maximum on any host. In fact, experiments by Gould (1979) and Fry (1990) support the trade-off hypothesis for $T$. urticae. In these experiments, laboratory populations that had been allowed to adapt to cucumber and tomato, respectively, lost a considerable proportion of that adaptation after being maintained on a third host, lima bean, for about 10 generations. Selection rather than genetic drift appeared to be the more likely explanation for this "reversion," because population sizes were on the order of 1,000 or more.

While the reversion in the above experiments gave evidence that genotypes that were relatively well adapted to cucumber or tomato were selected against on bean, neither Gould nor Fry could detect statistically significant differences between the cucumber or tomato-adapted line and the control line in survival or fecundity on bean. In Fry's (1990) study, a confidence interval showed that fecundity on bean of the tomatoadapted line was probably not more than $10 \%$ lower than that of the control line. The fitness comparisons on bean in both studies were conducted under un-

${ }^{1}$ Present address: Department of Genetics, Box 7614, North Carolina State University, Raleigh, NC 27695 USA. crowded conditions, and it is possible that fitness differences were more pronounced under the crowded conditions of the reversion lines. Nonetheless, the fact that neither Gould nor Fry could detect reduced fitness on bean as a consequence of adaptation to cucumber or tomato suggests that the "trade-offs" were asymmetric, with large improvements in fitness on the latter hosts being accompanied by relatively small reductions in fitness on bean. Models of the maintenance of genetic variation by environmental heterogeneity show, however, that the likelihood of genetic variation being maintained increases with both the strength and the symmetry of selection in the different environments (Levene, 1953; Dempster, 1955; Gillespie and Turelli, 1989). Therefore, more symmetric trade-offs, in which adaptation to one host is accompanied by a large reduction in fitness on a second, might be more likely to maintain genetic variation than those observed by Gould and Fry.

One reason that the "trade-offs" observed by Gould and Fry were asymmetric may be that lima bean, in contrast to cucumber and tomato, is a relatively "benign" host for T. urticae. Hatching-to-adult survival of populations on this host is usually near $100 \%$ under uncrowded conditions (Lehr and Smith, 1957; Fry, 1990; the 8\% mortality reported in Fry, 1990 was mostly due to inviable eggs), suggesting that development on bean offers no special challenges to the mites. Thus, it might be unrealistic to expect a large reduction in survival on bean as a consequence of adaptation to other hosts. Strong, symmetric trade-offs might be more likely among hosts known to be able to cause considerable mortality to mites (henceforth, "stressful" hosts), and on which inter- and intrapopulation genetic variation in survival is known to be present.

It is also possible, however, that attaining high fitness on different "stressful" hosts often requires similar physiological or morphological adaptations in the mites. This is suggested by the work of Gould (1979), who showed that adaptation to cucumber was accompanied by an increase in survival on two other hosts causing high mortality of mites, tobacco and potato. Further work (Gould et al., 1982) showed that the cucumberadapted line was also better able to tolerate certain pesticides than the control line, indicating that the increased fitness on cucumber may have been the result of increased activity of a broad-spectrum mechanism for resisting the effects of toxins. If survival on different "stressful" hosts indeed often requires similar physiological abilities, then one would expect that adaptation to one "stressful" host would generally result in 
increased rather than decreased fitness on others. "Trade-offs" might then be manifested only as a slight reduction in fitness on relatively "benign" hosts like lima bean as a consequence of adaptation to "stressful" hosts.

I investigated how adaptation to tomato in a $\mathrm{Te}$ tranychus urticae population affected fitness on two other hosts, cucumber and tobacco. The latter hosts were chosen because they are known to be capable of causing considerable juvenile mortality of mites, and because genetic variation for survival on them is present in some $T$. urticae populations (Gould, 1979); extensive among-population variation for survival on cucumber has also been documented (Gould, 1978b). The results rule out the possibility of symmetric trade-offs between adaptation to tomato and adaptation to cucumber and tobacco in the population I studied. While some trends towards increased fitness of the tomatoadapted line on cucumber and tobacco were detected, overall the results indicate that adaptation to tomato proceeded largely independently of adaptation to cucumber and tobacco.

\section{MATERIALS AND METHODS}

For this work, I made use of the tomato-adapted population (the "tomato line") and two control populations (the "bean" and "minimum selection" lines) described in Fry $(1989,1990)$. All three lines were established from the same, recently collected base population; the tomato line was maintained on potted tomato plants, while the bean and minimum selection lines were maintained on lima bean. The minimum selection line was originally intended as the control for the other two lines, being maintained in a manner designed to minimize its adaptation to the laboratory environment containing bean (for details, see Fry, 1989). The bean line, however, showed no evidence for divergence from the minimum selection line in any measure of fitness on bean, tomato, or three other hosts (Fry, 1988b, 1989, this paper), and thus can also be viewed as a control for evolutionary changes in the tomato line. Twenty-five weeks (ca 8 generations) after the lines were established, juvenile survival on tomato of tomato line mites was $56 \%$ compared to $25 \%$ in the two control lines, and development on tomato was also faster in the tomato line (Fry, 1989).

Twenty-five to 40 weeks after establishment of the lines, I compared the tomato line to both control lines in juvenile survival and development rate on tobacco (cv "Havana 501"), and to the minimum selection line only in survival, development rate, and fecundity on cucumber (cv "Hawaii 60G-10"). To compare lines in survival and development rate, I used a randomizedblock design, in which each block consisted of separate cohorts of mites from the two or three lines being compared, reared on the same plant at the same time. To initiate a block, I created two or three circular enclosures made of tanglefoot, a sticky substance that entraps mites; enclosures on tobacco $(2.5 \mathrm{~cm}$ diameter) were on the same leaf of 7-8 week old plants, while enclosures on cucumber ( $2 \mathrm{~cm}$ diameter) were on opposite cotyledons of 3-4 week old seedlings. Four or five (tobacco) or two (cucumber) adult females from the appropriate line were introduced to each enclosure, allowed to lay eggs for one day, and then removed. To minimize variation caused by parental environment, all females used to lay eggs had been reared on lima bean, regardless of source line. The progeny obtained in this way $(\bar{x}$, range: $31,3-67$ on tobacco; $14,3-30$ on cucumber) were allowed to develop until ca $50 \%$ had reached the adult stage; the development time required was 9-13 days on tobacco and 9 days on cucumber (within a block, all lines were given the same amount of time to develop). After the development period, the progeny were examined under a dissecting microscope and classified as live adults, live juveniles, or dead. Full details of these tests are presented elsewhere (Fry, 1988b, 1989).

For statistical analysis, I calculated the proportion of mites per enclosure that were alive, and the proportion of live mites that had reached the adult stage. The latter proportion served as a qualitative index of development rate [the tomato and control lines were strongly differentiated in this measure on tomato (Fry, 1989)]. Mites that had become caught in the tanglefoot barriers (about 15\% on average) were excluded; this did not affect the outcome of any analysis. Angulartransformed proportions were compared among lines by means of two-way unreplicated analysis of variance. I also constructed $95 \%$ confidence intervals for the difference in mean proportion surviving between lines on both hosts (because the development rate measure was a relative one, similar confidence intervals for development rate would have little meaning). On cucumber, the confidence interval was obtained by first calculating the difference in untransformed survival between the tomato and minimum selection lines for each block; because these differences were approximately normally distributed (Shapiro-Wilk test, $P>0.4$ ), the ordinary parametric confidence interval for the mean of the distribution of differences could be used. On tobacco, differences calculated similarly were not normally distributed; instead, I calculated a confidence interval in angular-transformed survival units for the linear contrast between the tomato line and the mean of the bean and minimum selection lines (Neter et al., 1990). While the confidence limits so obtained cannot be back-transformed into differences in the untransformed proportions surviving, they can be used as a rough gauge of the magnitude of any possible differences. I note that the proportion surviving in these tests was not correlated with the number of mites per enclosure (Spearman rank correlations, $P>0.05$ in each case).

To compare the tomato and minimum selection lines in fecundity on cucumber, I placed single bean-reared adult females, two days past emergence, inside tanglefoot enclosures on cucumber seedlings. The total number of eggs produced over a 12-day period was recorded, with the females being transferred to a fresh seedling after the sixth day. The resulting fecundity distributions were strongly skewed to the left and leptokurtic; accordingly, I used the Mann-Whitney $U$ test to compare lines.

\section{RESULTS}

There was no significant variation among the tomato, bean, and minimum selection lines in survival on tobacco, although a slight trend towards higher survival of the tomato line was present (Tables 1 and 2). The $95 \%$ confidence interval for the linear contrast between the mean of the tomato line and the mean of 
TABLE 1. Mean proportions of mites surviving and proportions of live mites that had reached the adult stage (a measure of development rate) in tests on tobacco and cucumber. M.S. line = Minimum selection line.

\begin{tabular}{ccccccccc}
\hline \hline & \multicolumn{3}{c}{ Mean proportion surviving } & & \multicolumn{3}{c}{ Mean proportion adults } \\
\cline { 2 - 4 } Host & Tomato line & Bean line & M.S. line & & Tomato line & Bean line & M.S. line \\
\hline Tobacco & 0.93 & 0.91 & 0.90 & & 0.50 & 0.45 & 0.49 \\
Cucumber & 0.74 & - & 0.75 & & 0.47 & - & 0.44 \\
\hline
\end{tabular}

the two control lines shows that angular-transformed survival of the tomato line may have been less than that of the control lines by 0.022 radian, or greater than that of the control lines by 0.133 radian. These lower and upper limits are $1.7 \%$ and $9.9 \%$, respectively, of the grand mean survival of 1.336 radians. Therefore, adaptation to tomato in the tomato line may have substantially increased ability to survive on tobacco, but did not substantially decrease it.

The tomato and minimum selection lines did not differ significantly in survival on cucumber (Tables 1 and 2 ). The $95 \%$ confidence interval for the mean difference in the untransformed proportions surviving shows that the true difference (tomato line survival minus minimum selection line survival) was probably between -0.06 and +0.05 , compared to the grand mean survival of 0.75 . Thus, adaptation to tomato did not substantially alter ability to survive on cucumber.

There was no variation among lines in development rate on either host, although a nonsignificant trend towards faster development rate in the tomato line was present on cucumber (Tables 1 and 2). Similarly, the tomato and minimum selection lines did not differ significantly in fecundity on cucumber (Mann-Whitney $U=351, P=0.20)$; means for the two lines were 68.6 $(\mathrm{SD}=16.2, N=29)$ and $64.7(\mathrm{SD}=16.0, N=30)$, respectively. A nonparametric $95 \%$ confidence interval for the difference in means between the two lines (Conover, 1980) indicates that the tomato line may have had lower fecundity than the minimum selection line by 2 eggs, or higher fecundity by 10 eggs, with these differences corresponding to $3 \%$ and $15 \%$ of the grand mean, respectively. Therefore, adaptation to tomato did not greatly decrease fecundity on cucumber, but a substantial increase cannot be ruled out.

\section{Discussion}

The results presented here show that adaptation to tomato in a Tetranychus urticae population did not greatly reduce fitness on two other hosts that can cause appreciable juvenile mortality of mites and on which genetic variation for fitness is present in some populations. Because a large increase in fitness on tomato was not accompanied by a large decrease in fitness on tobacco or cucumber, the possibility of symmetric tradeoffs among these hosts can be ruled out for the particular population studied. In contrast, it was not possible to rule out a substantial increase in fitness on tobacco or cucumber as a consequence of adaptation to tomato. There are two reasons, however, to suspect that the slight trends towards higher fitness of the tomato line than the control lines on these hosts did not reflect real differences among the lines. First, the trends were not consistent among the different fitness components on each host. Second, although the most nearly statistically significant difference was between the tomato and minimum selection lines in development rate on cucumber (Table 2), Gould (1979) did not observe an increase in development rate on cucumber even when mites were selected on this host, making such an increase as a correlated response to selection on tomato seem a priori unlikely. These arguments indicate that the most parsimonious interpretation of the results of this study is that loci affecting fitness on tomato were close to neutral on tobacco and cucumber.

Gould (1979) and Fry (1990, this paper) have sought possible trade-offs in fitness among seven pairs of hosts in $T$. urticae; in no case was a pattern of symmetric trade-offs found. It would be premature, however, to conclude that nearly symmetric trade-offs do not occur in $T$. urticae. Not only is the number of hosts so far examined small in relation to the host range of $T$. urticae, but certain broad categories of hosts, including woody plants and grasses, were not examined. In addition, experiments of Jesiotr and Suski (1976) and Jesiotr (1979) gave suggestive evidence that rearing mites on bean for several generations severely reduced fitness on rose, and vice versa.

Nonetheless, because clear evidence for symmetric trade-offs has not been found in $T$. urticae, it is important to consider mechanisms that might maintain genetic variation for fitness on particular hosts in their

TABLE 2. Results of two-way unreplicated analyses of variance on angular-transformed proportions surviving and proportions of live mites that had reached the adult stage. All mean squares have been multiplied by $10^{4}$.

\begin{tabular}{|c|c|c|c|c|c|c|}
\hline \multirow[b]{2}{*}{ Host } & \multirow[b]{2}{*}{ Character } & \multicolumn{3}{|c|}{ Mean squares $(d f)$} & \multirow[b]{2}{*}{$F$} & \multirow[b]{2}{*}{$P$} \\
\hline & & Lines & Blocks & Error & & \\
\hline Tobacco & Proportion live & $397(2)$ & $469(35)$ & $362(70)$ & 1.10 & $0.34^{1}$ \\
\hline Cucumber & Proportion live & $21(1)$ & $2,314(70)$ & $831(70)$ & 0.02 & 0.88 \\
\hline Tobacco & Proportion adults & $418(2)$ & $4,683(41)$ & $436(82)$ & 0.96 & $0.39^{2}$ \\
\hline Cucumber & Proportion adults & $1,872(1)$ & $1,728(68)$ & $657(68)$ & 2.85 & 0.10 \\
\hline
\end{tabular}

${ }^{1}$ Single degree-of-freedom contrasts: Bean line versus minimum selection line, $F=0.17, P=0.68$; Tomato line versus bean and minimum selection Single degree-of-freedo
lines: $F=2.02, P=0.16$.

lines: $F=2.02, P=0.16$. lines: $F=0.44, P=0.51$. 
absence. The asymmetry of the trade-offs observed by Fry (1990) between fitness on tomato and fitness on bean suggests that selection on tomato would overwhelm selection on bean in an environment consisting of equal frequencies of the two hosts. The host range of T. urticae, however, includes hundreds of host species, and most populations probably use many hosts in a year (e.g., Brandenburg and Kennedy, 1981, 1982). This means that the entire pattern of genetic variation and covariation in fitness among large sets of hosts needs to be considered. If, for example, genotypes favored on tomato are weakly selected against not only on bean but on many other hosts, then occasional strong selection on tomato could be counterbalanced by weaker but more frequent selection on the other hosts. The possibility of this balance being achieved would be enhanced if, as the results of this study suggest, fitness on tomato was not strongly positively correlated with fitness on other "stressful" hosts such as cucumber. Fry (1990) discusses other factors that may influence whether trade-offs in fitness on different hosts could maintain genetic variation in $T$. urticae.

Of course, it is possible that mechanisms not involving trade-offs play a role in maintaining genetic variation for fitness on particular hosts in $T$. urticae. The possibility that $T$. urticae populations are not at equilibrium for fitness on the hosts they use cannot be ruled out. Under this hypothesis, genetic variation for fitness on particular hosts would eventually disappear if current patterns of host utilization were continued for long enough. Another possibility is that mutation contributes significantly to genetic variation for fitness on some hosts (cf Mitter and Futuyma, 1983; Charlesworth, 1987). The role of mutation could be particularly important in cases where loci under strong selection on a host are under much weaker selection on others, as appears to be the case for tomato. If a population were to use tomato only $2 \%$ of the time, for example, the net effect of selection against mutations lowering fitness on tomato would probably be weak, and substantial genetic variance for fitness on tomato might be permanently maintained as a result. This mechanism in not inconsistent with the simultaneous operation of weak trade-offs to preserve genetic variation.

\section{ACKNOWLEDGMENTS}

I thank D. Goldberg, F. Gould, P. Grant, M. Martin, B. Rathcke, and P. Smouse for their advice and encouragement. D. Goldberg, D. Karowe, B. Rathcke, M. Rausher, P. Smouse, and the anonymous reviewers made valuable comments on earlier versions of the manuscript. The work was supported by grants from the University of Michigan and Sigma Xi. The Matthaei Botanical Gardens of the University of Michigan provided greenhouse space and materials for growing plants. This paper is based on research conducted for my Ph.D. dissertation.

\section{LITERATURE CITED}

BRANDENBURG, R. L., AND G. G. KENNEDY. 1981. Overwintering of the pathogen Entomophthora floridana and its host, the twospotted spider mite. J. Econ. Entomol. 74:428-431.

—. 1982. Intercrop relationships and spider mite dispersal in a corn/peanut agro-ecosystem. Entomol. Exp. Appl. 32:269-276.

Charlesworth, B. 1987. The heritability of fitness, pp. 21-40. In J. W. Bradbury and M. B. Andersson (eds.), Sexual Selection: Testing the Alternatives. Wiley, N.Y., USA.

Conover, W. J. 1980. Practical Nonparametric Statistics, 2nd ed. Wiley, N.Y., USA.

DEMPSTER, E. R. 1955. Maintenance of genetic heterogeneity. Cold Spring Harbor Symp. Quant. Biol. 20:25-32.

FRY, J. D. 1988a. Variation among populations of the twospotted spider mite, Tetranychus urticae Koch (Acari: Tetranychidae), in measures of fitness and host-acceptance behavior on tomato. Environ. Entomol. 17:287-292.

- $1988 b$. Genetic variation and genetic correlations in host-specific fitness in the phytophagous mite Tetranychus urticae Koch. University of Michigan, Ann Arbor. University Microfilms International, Ann Arbor, MI. Ph.D. Dissertation.

- 1989. Evolutionary adaptation to host plants in a laboratory population of the phytophagous mite Tetranychus urticae Koch. Oecologia 81:559-565.

1990. Trade-offs in fitness on different hosts: Evidence from a selection experiment with a phytophagous mite. Am. Nat. 136:569-580.

FutuYMa, D. J., AND S. C. Peterson. 1985. Genetic variation in the use of resources by insects. Annu. Rev. Entomol. 30:217-238.

Gilbert, J. C., J. T. ChinN, AND J. S. TANaka. 1966. Spider mite tolerance in multiple disease resistant tomatoes. Proc. Am. Soc. Hort. Sci. 89:559-562.

Gillespie, J. H., AND M. Turelui. 1989. Genotypeenvironment interactions and the maintenance of polygenic variation. Genetics 121:129-138.

Gould, F. 1978a. Resistance of cucumber varieties to Tetranychus urticae: Genetic and environmental determinants. J. Econ. Entomol. 71:680-683.

- $1978 \mathrm{~b}$. Predicting the future resistance of crop varieties to pest populations: A case study of mites and cucumbers. Environ. Entomol. 7:622-626.

- 1979. Rapid host range evolution in a population of the phytophagous mite Tetranychus urticae Koch. Evolution 33:791-802.

Gould, F., C. R. CARroll, AND D. J. Futumma. 1982. Cross-resistance to pesticides and plant defenses: $A$ study of the twospotted spider mite. Entomol. Exp. Appl. 31:175-180.

Jeppson, L. R., H. H. Keifer, AND E. W. BAKer. 1975. Mites Injurious to Economic Plants. University of California Press, Berkeley, CA, USA.

Jesiotr, L. J. 1979. The influence of the host plants on the reproduction potential of the twospotted spider mite, Tetranychus urticae Koch (Acarina: Tetranychidae). II. Responses of the field population feeding on roses and beans. Ekol. Polska 27:351355.

Jesiotr, L. J., AND Z. W. Suski. 1976. The influence of the host plants on the reproduction potential of the twospotted spider mite, Tetranychus urticae Koch (Acarina: Tetranychidae). Ekol. Polska 24: 407-411.

Lehr, R., AND F. F. Smith. 1957. The reproductive capacity of three strains of the two-spotted spider mite complex. J. Econ. Entomol. 50:634-636. 
LEVENE, H. 1953. Genetic equilibrium when more than one ecological niche is available. Am. Nat. 87: 331-333.

MrtTer, C., AND D. J. FutuYMa. 1983. An evolutionary-genetic view of host-plant utilization by insects, pp. 427-459. In R. F. Denno and M. S. McClure (eds.), Variable Plants and Herbivores in Natural and Managed Systems. Academic Press, N.Y., USA.

Neter, J., W. Wasserman, AND M. H. KutNeR. 1990.
Applied Linear Statistical Models, 3rd ed. Irwin, Homewood, IL, USA.

RAUSHER, M. D. 1983. Ecology of host-selection behavior in phytophagous insects, pp. 223-257. In R. F. Denno and M. S. McClure (eds.), Variable Plants and Herbivores in Natural and Managed Systems. Academic Press, N.Y., USA.

Corresponding Editor: T. Mackay 